\title{
TESTE RÁPIDO DE CONDUTIVIDADE ELÉTRICA E CORRELAÇÃO COM OUTROS TESTES DE VIGOR ${ }^{1}$
}

\author{
LUZINEIDE F. DE CARVALHO2, CARLOS SIGUEYUKI SEDIYAMA ${ }^{3}$, \\ DENISE CUNHA FERNANDES DOS SANTOS DIAS ${ }^{4}$, MÚCIO SILVA REIS ${ }^{4}$, MAURÍLIO ALVES MOREIRA ${ }^{3}$
}

\begin{abstract}
RESUMO - O presente trabalho teve como objetivo estimar a correlação entre os resultados de condutividade elétrica realizado em diferentes temperaturas e períodos de embebição com outros testes de qualidade fisiológica. Utilizaram-se sementes de soja colhidas no estádio R8 e aos 15, 30 e 45 dias após R8, nos anos agrícolas 2001/2002 e 2002/2003. A qualidade foi avaliada pelos testes de germinação, primeira contagem da germinação, envelhecimento acelerado, emergência em leito de areia e condutividade elétrica. No teste de condutividade elétrica, além da metodologia tradicional, na temperatura de $25^{\circ} \mathrm{C}$ por 24 horas, estudaram-se outras metodologias, utilizando 20 subamostras de 10 sementes mantidas em temperaturas de $32^{\circ} \mathrm{C}$ ou de $40^{\circ} \mathrm{C}$ por $10,30,60$ e 120 minutos. As metodologias estudadas correlacionaram-se bem com a metodologia tradicional, como também, com os outros testes avaliados e o período de 120 minutos, em ambas as temperaturas, foi o que melhor correlacionou com os outros testes avaliados, equiparando-se ao período de 24 horas.
\end{abstract}

Termos para indexação: Glycine max, correlação, temperatura e período de embebição.

\section{FAST ELECTRICAL CONDUCTIVITY TEST AND CORRELATION WITH OTHER VIGOR TESTS}

\begin{abstract}
The objective of this study was to estimate the correlation between the results of the electric conductivity test carried out with different temperatures and soaking periods and other tests that estimate seed physiological quality. Soybean seeds were harvested at the R8 stage and 15, 30 and 45 days after R8, in the 2001/2002 and 2002/2003 growing seasons. Seed quality was evaluated by the germination, first germination count, accelerated aging, sand bed emergence and electric conductivity tests. In addition to the traditional electric conductivity test, at $25^{\circ} \mathrm{C}$ for 24 hours, other methodologies were evaluated, using 20 subsamples of 10 seeds kept at $32^{\circ} \mathrm{C}$ or $40^{\circ} \mathrm{C}$ for $10,30,60$ and 120 minutes. The studied methodologies correlated well with the traditional methodology, as well as with the other tests evaluated and the 120-min period, at both temperatures, correlated best with the other evaluated tests, being equivalent to the 24-h period.
\end{abstract}

Index terms: Glycine max, correlation, temperature and soaking period.

${ }^{1}$ Submetido em 25/07/2008. Aceito para publicação em 28/01/2009 Parte
da Tese de Doutorado da primeira autora apresentada a UFV.

${ }^{2}$ Eng. Agr., D.Sc., bolsista-DCR, Embrapa Meio-Norte. Teresina, PI, Brasil. CEP 64006-220. luzineidecarvalho@yahoo.com.br.
${ }^{3}$ Eng. Agr., Ph.D., Professor Titular, Dep. de Fitotecnia, UFV. CEP 36571000 Viçosa, MG, Brasil. csediyama@ufv.br.

${ }^{4}$ Eng. Agr., D.Sc., Professor Titular, Dep. de Fitotecnia, UFV. CEP 36571000 Viçosa, MG, Brasil.msreis@ufv.br; dcdias@ufv.br. 


\section{INTRODUÇÃO}

O teste de condutividade elétrica tem sido considerado por pesquisadores como um bom indicador da emergência de plântulas em campo. Marcos Filho et al. (1986) e Rech et al. (1999), entre outros, demonstraram correlação altamente significativa entre essas características para sementes de ervilha.

Dos estudos iniciais com sementes de ervilha, várias pesquisas, envolvendo a condutividade elétrica, têm sido conduzidas como método para adaptar seu uso para outras espécies. O teste de condutividade elétrica aplicado em sementes de soja foi também considerado um indicador mais eficiente da emergência de plântula em campo do que o teste de germinação (Oliveira et al., 1984).

Estudos com sementes de soja colhidas em diferentes épocas e armazenadas em ambientes distintos demonstraram que o teste de condutividade forneceu informações mais precisas quanto à qualidade fisiológica do que o teste de germinação, envelhecimento acelerado (Marcos Filho et al., 1986). Prete et al. (1994) detectaram correlação altamente significativa entre os resultados obtidos pelo teste de condutividade elétrica e os de emergência de plântulas de soja em campo. O teste foi, então, suficiente para classificar lotes de sementes e cultivares dentro de níveis de vigor.

Mais recentemente, Vieira et al. (1999a), trabalhando com sementes de soja, após as avaliações de qualidade e experimentos de emergência em campo concluíram que o teste de condutividade elétrica, por meio do uso de faixas de valores, permite estimar, com alto grau de precisão, o desempenho de lotes em campo, dependendo das condições climáticas presentes no momento da semeadura. Resultado semelhante foi encontrado por Fagioli (1997) com sementes de milho.

A maioria dos trabalhos concentra estudo sobre a relação dos testes de condutividade e a emergência de plântulas em campo, sendo poucos aqueles que correlacionam os resultados de condutividade com outros testes de vigor. Nesse sentido, Loeffler et al. (1988) observaram correlação com outros testes de avaliação de vigor como primeira contagem, velocidade de germinação e envelhecimento acelerado, resultados similares aos de Vieira et al. (1999b), que apontaram boa correlação entre os resultados obtidos pelo teste de condutividade elétrica com os de emergência de plântulas em campo, germinação e envelhecimento acelerado. Do mesmo modo, Santos (2004) verificou correlação significativa entre os resultados dos testes de condutividade elétrica e os de germinação.

Ayala-Aguilera e Menezes (2000) constataram que os testes de germinação, envelhecimento acelerado e condutividade elétrica são bons indicadores para selecionar cultivares de soja em relação à qualidade das sementes. Resultados semelhantes foram encontrados por Salinas et al. (2001), em estudo comparativo entre os testes de vigor para avaliação da qualidade de sementes de soja, tendo verificado que o teste de condutividade elétrica é considerado bom para detectar as alterações nas membranas citoplasmáticas, em estádios precoces de deterioração.

Entre as inúmeras vantagens do teste de condutividade elétrica como um bom indicador do vigor de sementes está, ainda, o fato de ele ser considerado um teste rápido por estar relacionado com eventos iniciais da sequência de deterioração das sementes, como a degradação das membranas celulares e a redução das atividades respiratórias e biossintéticas (Delouche e Baskin, 1973). O teste de condutividade elétrica avalia o grau de estruturação das membranas celulares em decorrência da deterioração das sementes, por meio da determinação da quantidade de íons lixiviados em solução de embebição. As sementes de menor potencial fisiológico liberam maior quantidade de lixiviados, como consequência da menor estruturação e seletividade das membranas (Vieira e Krzyzanowski, 1999).

Vários fatores relacionados às características da semente ou da própria metodologia empregada podem interferir nos resultados e, conseqüentemente, na interpretação do teste (Vieira e Krzyzanowski, 1999). Entre esses fatores, está o período (Loeffler et al., 1988; Marcos Filho et al., 1990; Dias e Marcos Filho, 1996; Vanzolini e Nakagawa, 1999) e temperatura de embebição (Leopold, 1980; Gaspar e Nakagawa, 2002), os quais podem causar aumento e, ou, redução significativa na condutividade elétrica da solução de embebição das sementes.

Tradicionalmente, o teste de condutividade elétrica tem sido realizado com 24 horas de condicionamento, período esse recomendado pela International Seed Testing Association (ISTA) (Matthews e Powell, 1981), Association of Official Seed Analysts (AOSA, 1983) e por Vieira (1994) como o adequado para rotina normal dos laboratórios de análise de sementes. No entanto, várias pesquisas (Loeffler et al., 1988; Marcos Filho et al., 1990; Dias e Marcos Filho, 1996) têm apontado para a possibilidade de redução desse período, uma vez que períodos mais curtos têm sido capazes de discriminar lotes de sementes de soja com níveis distintos de vigor.

A temperatura de embebição influencia diretamente a velocidade de embebição e a lixiviação de eletrólitos do interior das células para o meio externo (Leopold, 1980; 
Murphy e Noland, 1982). Segundo Murphy e Noland (1982), esse efeito da temperatura pode estar relacionado com a alteração na viscosidade da água. $\mathrm{O}$ efeito da temperatura sobre a embebição e lixiviação ocorre, basicamente, sobre a quantidade e velocidade de perda de lixiviados (Vieira e Krzyzanowski, 1999).

Assim, o presente trabalho teve como objetivo estimar a correlação dos resultados obtidos pelo teste de condutividade elétrica realizado em diferentes temperaturas e períodos de embebição com outros testes da qualidade fisiológica de sementes de soja.

\section{MATERIAL E MÉTODOS}

As sementes utilizadas no experimento foram multiplicadas em campos experimentais Professor Diogo Alves de Mello da Universidade Federal de Viçosa-UFV nos anos agrícolas 2001/2002 e 2002/2003. No primeiro ano multiplicaram-se sementes das cultivares Cristalina, DokoRC, CAC-1 e Emgopa 312 e no segundo multiplicaram-se sementes dessas mesmas cultivares e das variedades triplonulas (ausência de lipoxigenase nas sementes): UFV TN-102, derivada das cultivares CAC-1 e UFV TN-104 originadas da cultivar Cristalina.

O ensaio foi constituído de dois blocos com quatro parcelas no primeiro ano e três blocos com seis parcelas no segundo. As sementes foram colhidas manualmente no estádio de maturação R8 da escala de Fehr e Caviness (1977) e aos 15,30 e 45 dias após o estádio R8. Após a secagem, foi realizada a debulha das vagens em trilhadeira estacionária e as sementes foram armazenadas em câmara fria à temperatura de $10^{\circ} \mathrm{C}$ e umidade relativa $60 \%$ até o início das avaliações. Os testes de avaliação da qualidade fisiológica foram conduzidos no Laboratório de Análise de Sementes e casa de vegetação da Universidade Federal de Viçosa (UFV).

O teste de condutividade elétrica (CE) foi realizado de três maneiras, sendo que na primeira utilizou-se a metodologia tradicional, que consistiu de duas repetições de campo, com quatro subamostras de 50 sementes em cada repetição, pesadas com precisão de duas casas decimais e colocadas em copos plásticos $(200 \mathrm{~mL})$ contendo $75 \mathrm{~mL}$ de água destilada. Em seguida foram levadas para germinador na temperatura de $25^{\circ} \mathrm{C}$, em que permaneceram por $24 \mathrm{~h}$. Após esse período, os recipientes foram retirados (16 copos de cada vez) e, com um bastão, as soluções contendo as sementes foram levemente agitadas para uniformização dos lixiviados, e imediatamente procedeu-se à leitura em condutivímetro (Vieira, 1994). A segunda, metodologia proposta, cada repetição foi constituída de 20 subamostras de 10 sementes, colocadas em copos plásticos com $25 \mathrm{~mL}$ de água destilada e mantidos por 10, 30, 60 e 120 minutos em incubadora tipo BOD, na temperatura de $32^{\circ} \mathrm{C}$. Decorrido cada período de embebição, procedeu-se à leitura da condutividade elétrica da solução e, finalmente, a terceira, seguiu-se o mesmo procedimento da metodologia anterior, porém utilizando a temperatura de $40^{\circ} \mathrm{C}$. Nas três metodologias utilizaram-se o método de massa, com sementes não-selecionadas, conforme sugerido por Loeffler et al. (1988). Os resultados foram expressos em $\mu \mathrm{S} . \mathrm{cm}^{-1} \cdot \mathrm{g}^{-1}$ de sementes.

Os testes de germinação (TG) e de primeira contagem da germinação (PC) consistiram da utilização de quatro e duas subamostras de 50 sementes por tratamento, nos ensaios do primeiro e segundo ano, respectivamente. As sementes foram distribuídas sobre papel "germitest” previamente umedecido com água destilada, em quantidade equivalente a 2,5 vezes o seu peso seco. Os rolos confeccionados foram mantidos em germinador à temperatura de $25^{\circ} \mathrm{C} \pm 1^{\circ} \mathrm{C}$. As avaliações foram realizadas aos cinco dias (primeira contagem) e aos oito dias após a instalação do teste (contagem final). Foram determinadas as porcentagens de plântulas normais, anormais e sementes mortas, sendo os resultados expressos em porcentagem de plântulas normais, segundo os critérios estabelecidos pelas Regas para Análise de Sementes (Brasil, 1992).

Para o teste de envelhecimento acelerado (EA), foram utilizadas caixas plásticas (gerbox) contendo, ao fundo, $40 \mathrm{~mL}$ de água destilada, nas quais foram colocadas 200 e 100 sementes por tratamento, colhidas no primeiro (2001/2002) e segundo (2002/2003) ensaios de campo, respectivamente, sobre tela de aço, isolando as sementes da água líquida. As caixas foram mantidas em estufa incubadora tipo BOD à temperatura de $42^{\circ} \mathrm{C}$, durante $48 \mathrm{~h}$. (AOSA, 1983). Em seguida, instalou-se o teste de germinação, sendo a avaliação do número de plântulas normais feita aos cinco dias após a instalação do teste, expressando-se os resultados em porcentagem.

A emergência de plântulas em leito de areia (ELA) foi conduzida em casa de vegetação, utilizando-se bandejas plásticas $(27 \times 32 \times 6 \mathrm{~cm})$ contendo areia previamente lavada e tratada com brometo de metila. No primeiro ensaio foram semeadas cinco subamostras de 40 sementes por tratamento em cinco sulcos por bandeja; no segundo ensaio foram semeadas 100 sementes, quatro subamostras de 25 sementes por sulco, num total de quatro sulcos por bandeja. Durante a condução do teste, a temperatura foi registrada duas vezes ao dia (manhã e tarde), discriminando-se as temperaturas 
máximas e mínimas. Realizaram-se irrigações sempre que necessário. A avaliação e contagem das plântulas normais foram realizadas quando a maioria das plântulas apresentou o primeiro par de folhas unifolioladas completamente expandidas.

Os dados provenientes dos testes de germinação, primeira contagem da germinação, envelhecimento acelerado e emergência de plântulas em leito de areia foram transformados em arco-seno $(\sqrt{x / 100} \quad)$ e submetidos à análise de correlação simples (r) entre os dados de condutividade elétrica, separadamente para cada cultivar. A significância dos valores de $\mathrm{r}$ foi determinada pelo teste t em $5 \%$ e $1 \%$ de probabilidade.

\section{RESULTADOS E DISCUSSÃO}

Os coeficientes de correlação simples referentes aos dados obtidos são apresentados nas Tabelas 1 (2001/2002) e $2(2002 / 2003)$.

Na Tabela 1 (primeiro ano), observou-se a existência de correlação significativa entre os dados de condutividade elétrica (24 horas a $25^{\circ} \mathrm{C}$ - metodologia tradicional) e germinação $(\mathrm{P} \leq 0,05)$, primeira contagem $(\mathrm{P} \leq 0,05)$, envelhecimento acelerado $(\mathrm{P} \leq 0,01)$ e emergência em leito de areia $(\mathrm{P} \leq 0,01)$, em todas as cultivares estudadas. Verificouse também correlação significativa $(\mathrm{P} \leq 0,01$ ou $\mathrm{P} \leq 0,05)$ entre os valores de condutividade (metodologia tradicional) e condutividade elétrica (metodologia proposta) conduzidas com 10, 30, 60 e 120 minutos nas temperaturas $32^{\circ} \mathrm{C}$ e $40^{\circ} \mathrm{C}$, em todas as cultivares estudadas, com exceção da cultivar CAC-1, que não apresentou correlação significativa no período de 60 minutos a $32^{\circ} \mathrm{C}$.

Esses resultados concordam com as observações de Loeffler et al. (1988), Vieira et al. (1998) com soja e Fagioli (1997) com milho, que constataram correlações significativas entre os valores de condutividade elétrica com os de germinação e envelhecimento acelerado. Santos (2004) também se referiram à existência de correlação significativa entre condutividade elétrica e germinação. Do mesmo modo, Vieira et al. (2001) verificaram que a baixa qualidade das sementes, obtidas no teste de envelhecimento acelerado, correlacionou-se com mudança na integridade das membranas medida por meio de teste de condutividade elétrica. Esse fato indica que aumentos nos valores de condutividade elétrica corresponderam à queda nos níveis de germinação e vigor das sementes, fato esse que concorda com observações na literatura, em que aumentos nos dados de condutividade elétrica correspondem à maior lixiviação de solutos e, portanto, à diminuição na qualidade fisiológica das sementes (Powell,1986; Marcos Filho et al., 1990; Sá, 1999).

As correlações da condutividade elétrica (metodologia proposta) realizada com $32^{\circ} \mathrm{C}$ e o teste de envelhecimento acelerado e o de emergência em leito de areia foram altamente significativas em todos os períodos de embebição das sementes das cultivares Doko-RC, Cristalina e Emgopa-312. A cultivar CAC-1, novamente, diferenciouse dos demais, apresentando correlação significativa apenas para os tempos de 30 e 120 minutos. Quanto à correlação entre os dados do teste realizado com $40^{\circ} \mathrm{C}$, notou-se que somente o período de 60 minutos (cultivar Cristalina) não se correlacionou com os dados de emergência em leito de areia, ou seja, todas as outras combinações se correlacionaram entre si em todas as cultivares, indicando uma tendência de variação semelhante entre a condutividade e cada teste correlacionado (Marcos Filho et al., 1987).

Os resultados de germinação e primeira contagem da germinação foram os que menos se correlacionaram com o teste de condutividade (metodologia proposta), exceto para a cultivar Cristalina que, em ambas as temperaturas, apresentou correlação altamente significativa entre os valores de condutividade elétrica, germinação e primeira contagem. Para a cultivar Doko-RC, na temperatura de $32^{\circ} \mathrm{C}$, também houve correlação significativa entre os testes aqui discutidos, com exceção do período de 30 minutos, que não se correlacionou com os testes supracitados. No entanto, na temperatura de $40^{\circ} \mathrm{C}$, notou-se correlação significativa somente a partir de 120 minutos, em ambos os testes. Já, nas cultivares Emgopa-312 e CAC-1 as correlações foram mais esparsas. Verificou-se que na cultivar Emgopa-312, na temperatura de $32^{\circ} \mathrm{C}$, houve correlação significava a partir de 60 minutos, enquanto a $40^{\circ} \mathrm{C}$, a partir de 30 minutos, os valores de condutividade se correlacionaram com os de germinação, e somente o período de 120 minutos se correlacionou com os valores da primeira contagem. Quanto a cultivar CAC-1, os resultados de condutividade a $32^{\circ} \mathrm{C}$ se correlacionaram significativamente com a germinação somente aos 120 minutos e com a primeira contagem, a partir de 30 minutos. A $40^{\circ} \mathrm{C}$, somente no período de 30 minutos não ocorreu correlação significativa entre os dados de condutividade, germinação e primeira contagem da germinação. Correlação menos acentuada entre esses três testes (condutividade, germinação e primeira contagem) era, de certa maneira, esperada, pois, estes avaliam diferentes aspectos da qualidade fisiológica das sementes. Rosa et al. (2000) observaram, em sementes de milho, correlação significativa entre o teste de 
TABELA 1. Coeficientes de correlação simples (r) entre os valores de condutividade elétrica (CE) com 20 subamostras e os testes de germinação (TG), primeira contagem de germinação (PC), envelhecimento acelerado (EA) e emergência em leito de areia (ELA), realizados com sementes de soja de quatro cultivares. Viçosa, MG, 2001/2002.

\begin{tabular}{|c|c|c|c|c|c|c|c|}
\hline \multirow{3}{*}{ Variedades } & \multirow{3}{*}{$\begin{array}{l}\text { Temp. } \\
\left({ }^{\circ} \mathrm{C}\right)\end{array}$} & \multirow{3}{*}{$\begin{array}{l}\text { Período de } \\
\text { Embebição }\end{array}$} & \multicolumn{5}{|c|}{ Coeficientes de Correlação } \\
\hline & & & CE 25 & TG & $\mathrm{PC}$ & EA & ELA \\
\hline & & & $\mu \mathrm{S} / \mathrm{cm} / \mathrm{g}$ & $\begin{array}{ll}----\cdot \\
-\end{array}$ & ---------- & -\%------- & ----------- \\
\hline \multirow{10}{*}{ Doko-RC } & $25^{\circ} \mathrm{C}$ & $\mathrm{CE}-24 \mathrm{~h}$ & - & $-0,7186^{*}$ & $-0,7242 *$ & $-0,8848 * *$ & $-0,8783 * *$ \\
\hline & \multirow{4}{*}{$32{ }^{\circ} \mathrm{C}$} & CE-10 min & $0,9085^{* *}$ & $-0,7307^{*}$ & $-0,7345^{*}$ & $-0,8673 * *$ & $-0,8759 * *$ \\
\hline & & CE-30 min & $0,9137 * *$ & $-0,7039$ & $-0,7074^{*}$ & $-0,8484 * *$ & $-0,8472 * *$ \\
\hline & & CE-60 min & $0,9145^{* *}$ & $-0,7251^{*}$ & $-0,7252 *$ & $-0,8594 * *$ & $-0,8679 * *$ \\
\hline & & CE-120 min & $0,9064 * *$ & $-0,7261^{*}$ & $-0,7657^{*}$ & $-0,8862 * *$ & $-0,8920 * *$ \\
\hline & \multirow{4}{*}{$40^{\circ} \mathrm{C}$} & CE-10 min & $0,9282 * *$ & $-0,6961$ & $-0,6943$ & $-0,8362 * *$ & $-0,8728 *$ \\
\hline & & CE-30 min & $0,9291 * *$ & $-0,6671$ & $-0,6571$ & $-0,8059 *$ & $-0,7939 *$ \\
\hline & & CE-60 min & $0,9294^{* *}$ & $-0,6466$ & $-0,6406$ & $-0,8013^{*}$ & $-0,7883^{*}$ \\
\hline & & CE-120 min & $0,9350 * *$ & $-0,7496^{*}$ & $-0,7440 *$ & $-0,8730 * *$ & $-0,8424^{*}$ \\
\hline & $25^{\circ} \mathrm{C}$ & $\mathrm{CE}-24 \mathrm{~h}$ & - & $-0,8425^{* *}$ & $-0,8381^{* *}$ & $-0,8923^{* *}$ & $-0,8284^{*}$ \\
\hline \multirow{8}{*}{ Cristalina } & \multirow{4}{*}{$32{ }^{\circ} \mathrm{C}$} & CE-10 min & $0,9330 * *$ & $-0,8442 * *$ & $-0,8244^{*}$ & $-0,8196 *$ & $-0,7369 *$ \\
\hline & & CE-30 min & $0,9767 * *$ & $-0,8714^{* *}$ & $-0,8633 * *$ & $-0,8564 * *$ & $-0,8002 * *$ \\
\hline & & CE-60 min & $0,9689 * *$ & $-0,8623^{* *}$ & $-0,8439 * *$ & $-0,8469 * *$ & $-0,7666^{* *}$ \\
\hline & & CE-120 min & $0,9320 * *$ & $-0,9509 * *$ & $-0,9355^{* *}$ & $-0,8928 * *$ & $-0,8474^{* *}$ \\
\hline & \multirow{4}{*}{$40{ }^{\circ} \mathrm{C}$} & CE-10 min & $0,9126^{* *}$ & $-0,9036^{* *}$ & $-0,8880 * *$ & $-0,7997^{*}$ & $-0,7591^{*}$ \\
\hline & & CE-30 min & $0,9583^{* *}$ & $-0,9003^{* *}$ & $-0,8816 * *$ & $-0,8489 * *$ & $-0,7851^{*}$ \\
\hline & & CE-60 min & $0,9134 * *$ & $-0,8542 * *$ & $-0,8375 * *$ & $-0,7587 *$ & $-0,7008$ \\
\hline & & CE-120 min & $0,9191^{* *}$ & $-0,9588 * *$ & $-0,9514 * *$ & $-0,8728 * *$ & $-0,8628 * *$ \\
\hline \multirow{9}{*}{ Emgopa-312 } & $25^{\circ} \mathrm{C}$ & $\mathrm{CE}-24 \mathrm{~h}$ & - & $-0,8304 *$ & $-0,8156^{*}$ & $-0,8353^{* *}$ & $-0,8296 *$ \\
\hline & \multirow{4}{*}{$32{ }^{\circ} \mathrm{C}$} & CE-10 min & $0,8232^{*}$ & $-0,6699$ & $-0,6458$ & $-0,8233^{*}$ & $-0,7666^{*}$ \\
\hline & & CE-30 min & $0,8115^{*}$ & $-0,6951$ & $-0,6786$ & $-0,8436 * *$ & $-0,7850 *$ \\
\hline & & CE-60 min & $0,8049 *$ & $-0,7890 *$ & $-0,7765^{*}$ & $-0,8958 * *$ & $-0,8569 * *$ \\
\hline & & CE-120 min & $0,8833^{* *}$ & $-0,8511^{* *}$ & $-0,8352 * *$ & $-0,9245 * *$ & $-0,8961 * *$ \\
\hline & \multirow{4}{*}{$40{ }^{\circ} \mathrm{C}$} & CE-10 min & $0,8858 * *$ & $-0,6789$ & $-0,6578$ & $-0,8161^{*}$ & $-0,7773 *$ \\
\hline & & CE-30 min & $0,9292 * *$ & $-0,7117^{*}$ & $-0,6889$ & $-0,8277^{*}$ & $-0,7847^{*}$ \\
\hline & & CE-60 min & $0,8704^{* *}$ & $-0,7115^{*}$ & $-0,6909$ & $-0,8189 *$ & $-0,7766^{*}$ \\
\hline & & CE-120 min & $0,9104^{* *}$ & $-0,7684^{*}$ & $-0,7481^{*}$ & $-08378 * *$ & $-0,7977^{*}$ \\
\hline
\end{tabular}




\begin{tabular}{|c|c|c|c|c|c|c|c|}
\hline \multirow{9}{*}{ CAC-1 } & $25^{\circ} \mathrm{C}$ & $\mathrm{CE}-24 \mathrm{~h}$ & - & $-0,8998 * *$ & $-0,8933^{* *}$ & $-0,9025^{* *}$ & $-0,9475 *$ \\
\hline & \multirow{4}{*}{$32{ }^{\circ} \mathrm{C}$} & CE-10 min & $0,7469 *$ & $-0,6420$ & $-0,6431$ & $-0,7040$ & $-0,6744$ \\
\hline & & CE-30 min & $0,7537^{*}$ & $-0,7063$ & $-0,7127^{*}$ & $-0,7666^{*}$ & $-0,7174^{*}$ \\
\hline & & CE-60 min & 0,6940 & $-0,7045$ & $-0,7156 *$ & $-0,6989$ & $-0,6161$ \\
\hline & & CE-120 min & $0,8566 * *$ & $-0,8887 * *$ & $-0,8940 * *$ & $-0,8847 * *$ & $-0,8034^{*}$ \\
\hline & \multirow{4}{*}{$40^{\circ} \mathrm{C}$} & CE-10 min & $0,8556 * *$ & $-0,7692 *$ & $-0,7693^{*}$ & $-0,9239 * *$ & $-0,8749 * *$ \\
\hline & & CE-30 min & $0,8314^{*}$ & $-0,6990$ & $-0,6971$ & $-0,9088 * *$ & $-0,8798 * *$ \\
\hline & & CE-60 min & $0,9089 * *$ & $-0,7577^{*}$ & $-0,7560 *$ & $-0,9144 * *$ & $-0,9185^{* *}$ \\
\hline & & CE-120 min & $0,9334 * *$ & $-0,7989 *$ & $-0,7970 *$ & $-0,9276 * *$ & $-0,9410^{* *}$ \\
\hline
\end{tabular}

** e * Significativos a $1 \%$ e $5 \%$, pelo teste de $\mathrm{F}$, respectivamente.

condutividade elétrica com o de germinação e envelhecimento acelerado a partir das oito horas de embebição e Marques et al. (2002), em sementes de jacarandá (condutividade entre primeira contagem, germinação e emergência em viveiro), a partir de 12 horas de embebição, nas temperaturas de 20 e $25^{\circ} \mathrm{C}$ e, na temperatura de $30^{\circ} \mathrm{C}$, observaram correlação significativa já com seis horas de embebição. Embora a referência tenha sido com sementes florestais, verificou-se certa similaridade entre esses resultados com os do presente trabalho, no sentido de que a utilização de temperaturas mais elevadas proporcionou correlações significativas entre os testes em períodos menores de embebição. Dias et al. (1996) destacaram correlação entre os resultados de emergência de campo e os de condutividade elétrica, observada a partir das 16 horas de embebição das sementes de soja.

De modo geral, as correlações entre os testes de condutividade a $25^{\circ} \mathrm{C} / 24$ horas, envelhecimento acelerado e emergência em leito de areia e a condutividade a $32^{\circ} \mathrm{C}$ e $40^{\circ} \mathrm{C}$ por 10, 30, 60 e 120 minutos, em diferentes tempos de embebição, foram as mais consistentes, indicando serem testes adequados para avaliar a qualidade das sementes. Em termos de cultivar, é importante notar que os valores obtidos para sementes da cultivar Cristalina apresentaram correlações altamente significativas entre todas as combinações estudadas, exceto entre o período de 60 minutos e emergência em leito de areia, evidenciando-se a alta correspondência entre os valores de condutividade elétrica na solução de embebição das sementes com a qualidade fisiológica dos lotes avaliados pelos outros testes empregados no presente trabalho. Assim, em todos esses testes, observou-se uma resposta diferencial em função do genótipo. Em algumas cultivares, obteve-se uma correlação altamente significativa e em outras não, devendo ressaltar que o efeito devido ao genótipo foi, em parte, responsável por esse comportamento, não devendo esquecer-se, entretanto, de que o ambiente teve também influência nesses resultados.

Analisando os dados obtidos no segundo ano (Tabela 2), as correlações entre os valores de condutividade (metodologia tradicional) e os testes de germinação, primeira contagem, envelhecimento acelerado e emergência em leito de areia foram altamente significativas $(\mathrm{P} \leq 0,01)$ em todos as cultivares, bem como entre o teste de condutividade a $32^{\circ} \mathrm{C}$ e $40^{\circ} \mathrm{C}$, exceto para a cultivar Cristalina, que aos $40^{\circ} \mathrm{C}$ se correlacionou, apenas, após os 120 minutos de embebição. Do mesmo modo, constataram-se correlações altamente significativas entre os valores de condutividade elétrica a $32^{\circ} \mathrm{C}$ e $40^{\circ} \mathrm{C}$ e os outros testes de avaliação da qualidade fisiológica (germinação, primeira contagem, envelhecimento acelerado e emergência em leito de areia), em todos os períodos de embebição, em quatro das seis cultivares estudadas.

Verificou-se, portanto, que as correlações entre os resultados de condutividade elétrica a $32^{\circ} \mathrm{C}$ e envelhecimento acelerado e a emergência de plântulas na cultivar Cristalina foram significativas a partir de 30 minutos, enquanto a $40^{\circ} \mathrm{C}$ não se observou correlação significativa. No teste de emergência de leito de areia, notou-se correlação entre o teste de condutividade a $32^{\circ} \mathrm{C}$ e a $40^{\circ} \mathrm{C}$ somente nos períodos de 30 e 60 minutos. Isso contraria as observações efetuadas anteriormente, pois, no primeiro ano e com essa cultivar, obteve-se correlação em quase todos os testes estudados. Essa observação indica que essa menor correspondência entre os dados seja, provavelmente, devida ao fato de o teste a $40^{\circ} \mathrm{C}$ ter sido diferente dos demais, no sentido de identificar a cultivar Cristalina como de melhor qualidade. Na cultivar Emgopa-312, constatou que o teste de envelhecimento acelerado não se correlacionou com os períodos de 60 e 120 minutos, a $32^{\circ} \mathrm{C}$; e com os de 10 e 30 minutos a $32^{\circ} \mathrm{C}$ e com todos os períodos a $40^{\circ} \mathrm{C}$. 
TABELA 2. Coeficientes de correlação simples (r) entre os valores de condutividade elétrica (CE) com 20 subamostras e os testes de germinação (TG), primeira contagem de germinação (PC), envelhecimento acelerado (EA) e emergência em leito de areia (ELA), realizados com sementes de soja de seis cultivares. Viçosa, MG, $2002 / 2003$

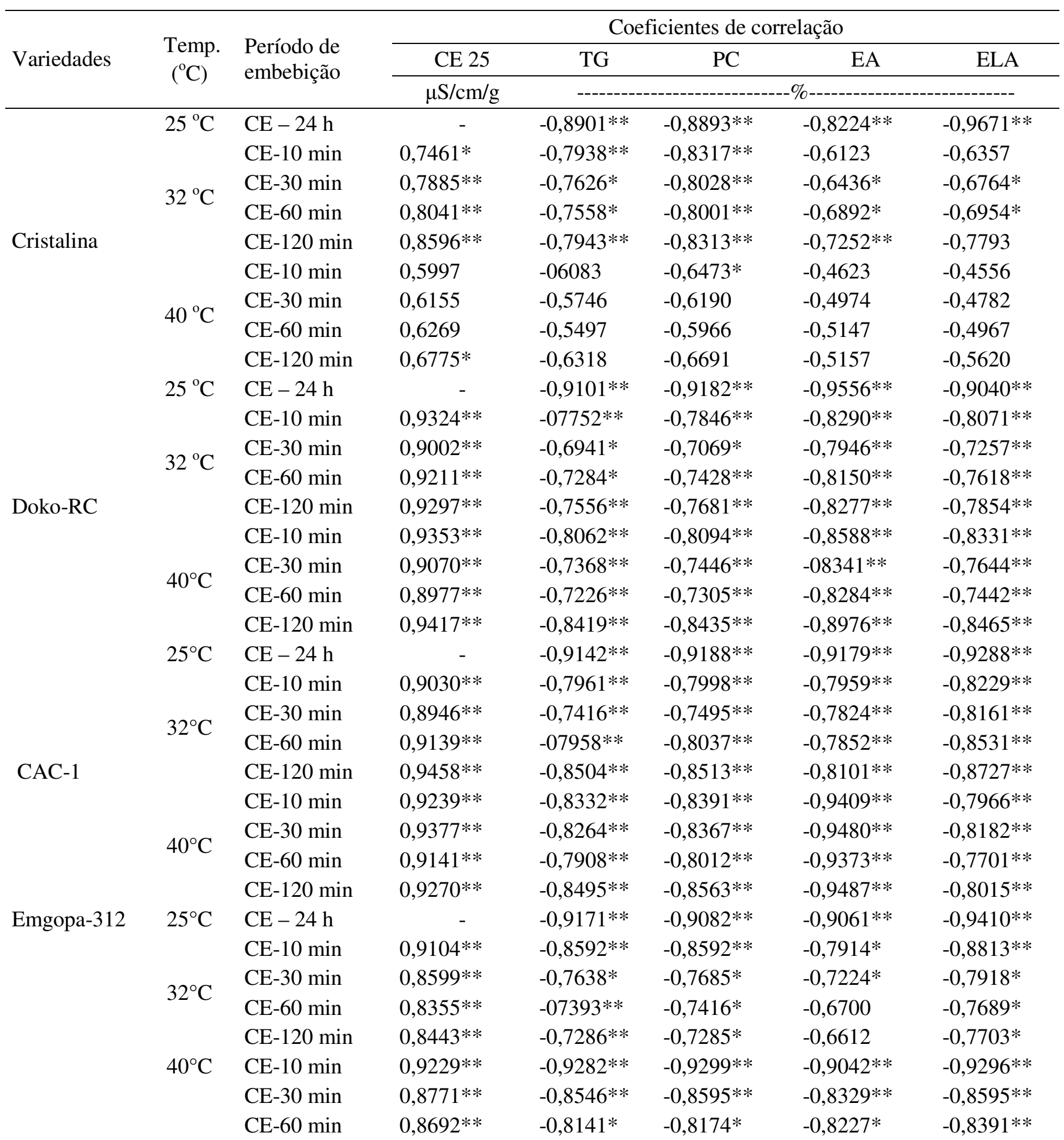




\begin{tabular}{|c|c|c|c|c|c|c|c|}
\hline & & CE-120 min & $0,9274 * *$ & $-0,8715^{* *}$ & $-0,8709 * *$ & $-0,8464 * *$ & $-0,8897 * *$ \\
\hline \multirow{9}{*}{ UFV-TN-102 } & $25^{\circ} \mathrm{C}$ & $\mathrm{CE}-24 \mathrm{~h}$ & - & $-0,9779 * *$ & $-0,9763 * *$ & $-0,9314 * *$ & $-0,9353^{* *}$ \\
\hline & \multirow{6}{*}{$32{ }^{\circ} \mathrm{C}$} & CE-10 min & $0,9304 * *$ & $-0,8773 * *$ & $-0,8893 * *$ & $-0,8574 * *$ & $-0,8273 * *$ \\
\hline & & CE-30 min & $0,9161 * *$ & $-0,8623 * *$ & $-0,8762 * *$ & $-0,8678 * *$ & $-0,8104 * *$ \\
\hline & & CE-60 min & $0,9187 * *$ & $-0,8625 * *$ & $-0,8778 * *$ & $-0,8479 * *$ & $-0,8490$ ** \\
\hline & & $\mathrm{CE}-120 \mathrm{~min}$ & $0,9613 * *$ & $-0,9164 * *$ & $-0,9255 * *$ & $-0,8717 * *$ & $-0,9045^{* *}$ \\
\hline & & CE-10 min & $0,9330 * *$ & $-0,8952 * *$ & $-0,9027 * *$ & $-0,8593 * *$ & $-0,8565^{* *}$ \\
\hline & & CE-30 min & $0,9473 * *$ & $-0,8992 * *$ & $-0,9054 * *$ & $-0,8773 * *$ & $-0,8720 * *$ \\
\hline & \multirow[t]{2}{*}{$40{ }^{\circ} \mathrm{C}$} & CE-60 min & $0,9511 * *$ & $-0,8987 * *$ & $-0,9014 * *$ & $-0,8846^{*}$ & $-0,8777 * *$ \\
\hline & & $\mathrm{CE}-120 \mathrm{~min}$ & $0,9483^{*}$ & $-0,8951 * *$ & $-0,8935 * *$ & $-0,8794 * *$ & $-0,8833$ ** \\
\hline \multirow{9}{*}{ UFV-TN-104 } & $25^{\circ} \mathrm{C}$ & $\mathrm{CE}-24 \mathrm{~h}$ & - & $-0,8624 * *$ & $-0,8539 * *$ & $-0,9329 * *$ & $-0,9011 * *$ \\
\hline & \multirow{4}{*}{$32{ }^{\circ} \mathrm{C}$} & CE-10 min & $0,8764 * *$ & $-0,7279 * *$ & $-0,7175 * *$ & $-0,8882 * *$ & $-0,8689 * *$ \\
\hline & & CE-30 min & $0,8664 * *$ & $-0,6927 *$ & $-0,6794 * *$ & $-0,8671 * *$ & $-0,8426 * *$ \\
\hline & & CE-60 min & $0,8741 * *$ & $-0,6902 *$ & $-0,6741^{*}$ & $-0,8706^{* *}$ & $-0,8390 * *$ \\
\hline & & CE-120 min & $0,9080 * *$ & $-0,7526 * *$ & $-0,7346 * *$ & $-0,9011 * *$ & $-0,9182 * *$ \\
\hline & \multirow{4}{*}{$40{ }^{\circ} \mathrm{C}$} & CE-10 min & $0,8252 * *$ & $-0,7088 * *$ & $-0,6973 *$ & $-0,8462 * *$ & $-0,8197 * *$ \\
\hline & & CE-30 min & $0,8252 * *$ & $-0,6827 *$ & $-0,6680 *$ & $-0,8492 * *$ & $-0,8290 * *$ \\
\hline & & CE-60 min & $0,8295^{* *}$ & $-0,6846^{*}$ & $-0,6661^{*}$ & $-0,8353 * *$ & $-0,8187 * *$ \\
\hline & & CE-120 min & $0,8649 * *$ & $-0,6956^{*}$ & $-0,6737^{*}$ & $-0,8525 * *$ & $-0,8662 * *$ \\
\hline
\end{tabular}

** e * Significativos a $1 \%$ e $5 \%$, pelo teste de $\mathrm{F}$, respectivamente.

É importante notar que, no segundo ano, as correlações entre os testes de avaliação da qualidade fisiológica e os de condutividade elétrica foram mais acentuadas do que as observadas no ano anterior, principalmente entre os testes de germinação e primeira contagem, cujo grau de associação com o teste de condutividade elétrica foi similar ao obtido nos demais testes. Isso permite supor que a deterioração das sementes em campo antes da colheita, conforme o ciclo das cultivares, por exemplo, poderia ter tido peso maior na qualidade final dos lotes produzidos naquele ano (2001/2002). Yaklich et al. (1979) e Martin et al. (1988) ressaltaram que os coeficientes de correlação variam entre anos e entre épocas de semeadura dentro do mesmo ano, o que pode explicar a diferença encontrada na cultivar Cristalina de um ano para o outro e a variação verificada nos coeficientes das outras características avaliadas (TG, PC, EA, ELA) nas duas etapas em que o trabalho foi desenvolvido.

Quanto à correlação entre os dados de condutividade e a emergência de plântulas em leito de areia, a literatura faz pouca referência e a maioria das publicações destaca correlações significativas entre condutividade e emergência em campo (Prete et al., 1994; Vieira et al., 1998 e 1999b; Fagioli, 1997). Entretanto, no presente trabalho a emergência em leito de areia resultou em correlações altamente significativas tanto nos dados da metodologia de condutividade elétrica tradicional quanto nos de metodologia proposta em ambos os anos.

Quando as correlações foram determinadas em função da cultivar, observou-se o mesmo tipo de associação (correlações negativas para CE x TG, CE x PC, CE x EA, e CE x ELA e positivas para CE- 25 x CE-32 e $40^{\circ} \mathrm{C}$ ), porém com grau de associação diferente, resultando em correlações não-significativas ou significativas em diferentes níveis de significância $(\mathrm{P} \leq 0,01$ ou $\mathrm{P} \leq 0,05)$. Isso evidencia a existência de resposta diferenciada conforme o genótipo (Yaklich et al., 1979; Panobianco e Vieira; 1996). Observou-se também que, numa mesma cultivar, os valores de "r" podem variar em função do ano, ocorrendo anos com correlações significativas ou não.

\section{CONCLUSÕES}

As metodologias de condutividade elétrica estudadas correlacionam-se com a metodologia tradicional e com os outros testes de vigor avaliados.

O período de 120 minutos de embebição das sementes no teste de condutividade elétrica, em temperaturas de $32^{\circ} \mathrm{C}$ ou de $40^{\circ} \mathrm{C}$, é o que melhor se correlacionou com os outros testes avaliados, equiparando-se ao período de 24 horas, tradicionalmente utilizado naquele teste. 


\section{REFERÊNCIAS}

AOSA. ASSOCIATION OF OFFICIAL SEED ANALYSTS. Seed vigor testing handbook. [S.l.]: AOSA, 1983. 93 p. (Contribution, 32).

AYALA-AGUILERA, L.; MENEZES, N.L. Seed quality characterization of soybean cultivars. Revista Brasileira de Agrociência, v.6, n.3, p.222-225, 2000.

BRASIL. Ministério da Agricultura e da Reforma Agrária. Regras para análise de sementes. Brasília, DF: SNDA/ DNDV/CLAV. 1992. 365p.

DELOUCHE, J.C.; BASKIN, C. Accelerated aging techniques for predicting the relative storability of seed lots. Seed Science and Technology, v.1, n.2, p.427-52, 1973.

DIAS, D.C.F.S.; MARCOS FILHO, J. Testes de condutividade elétrica para avaliação do vigor de sementes de soja (Glycine max (L.) Merrill). Scientia Agricola, v.53, n.1, p.1-11, 1996.

DIAS, D.C.F.S.; MARCOS-FILHO, J.; CARMELLO, Q.A.C. Potassium leakage test for the evaluation of vigour in soybean seeds. Seed Science and Technology, v.25, n. , p.7-18, 1996.

FAGIOLI, M. Relação entre condutividade elétrica e ou de sementes e a emergência de plântulas de milho em campo. 1997. 74f. Dissertação (Mestrado em Produção e Tecnologia de Sementes) - Universidade Estadual Paulista, Jaboticabal..

FEHR, W.R.; CAVINNESS, C.E. Stages of soybean development. Ames Iowa: Iowa State University: Cooperative Extension Service, 1977. 12p.

GASPAR, C.M.; NAKAGAWA, J. Teste de condutividade elétrica em função do período e da temperatura de embebição para sementes de milheto. Revista Brasileira de Sementes, v.24, n.2, p.82-89, 2002.

LEOPOLD, A.A. Temperature effects on soybean imbibition and leakage. Plant Physiology, v. 65, n. 4, p. 1096-8, 1980.

LOEFFLER, L.M.; TEKRONY, D.M.; EGLI, D.B. The bulk conductivity test as an indicator of soybean seed quality. Journal of Seed Technology, v.12, n.1, p.37-53, 1988.

MARCOS FILHO, J. CARVALHO, R.V.; CÍCERO, S.M.; DEMÉTRIO, C.G.B.. Qualidade fisiológica e comportamento de sementes de soja (Glycine max (L.) Merrill) no armazenamento e no campo. Anais Escola Superior de Agricultura "Luiz de Queiroz", v.43, p.389-443, 1986.

MARCOS FILHO, J.; CÍCERO, S.M.; SILVA, W.R. Avaliação da qualidade das sementes. Piracicaba: FEALQ,
1987. 230p.

MARCOS FLHO, J.; SILVA, W.R.; NOVEMBRE, A.C.; CHAMA, H. Estudo comparativo de métodos para a avaliação da qualidade fisiológica de sementes de soja, com ênfase ao teste de condutividade elétrica. Pesquisa Agropecuária Brasileira, v.25, n.12, p.1805-15, 1990.

MARQUES, M.A.; PAULA, R.C.; RODRIGUES, T.J.D. Adequação do teste de condutividade elétrica para determinar a qualidade fisiológica de sementes de jacarandá-dabahia (Dalbergia nigra (Vell.) Fr. All. ex Benth.). Revista Brasileira de Sementes, v.24, n.1, p.271-278, 2002.

MARTIN, B.A.; SMITH, S.O.; O’NELL, M. Relathionship between laboratory germination tests and field emergence of maize inbreds. Crop Science, v.28, n.5, p.801-5, 1988.

MATTHEWS, S.; POWELL, A.A. Electrical conductivity test. In: PERRY, D.A. (Ed.) . Handbook of vigour test methods. Zurich: ISTA, 1981. p.37-42

MURPHY, J.B.; NOLAND, T.L. Temperature effects on seed imbibition and leakage mediated by viscosity and membranes. Plant Physiology, v.69, n.2, p.428-431, 1982.

OLIVEIRA, M. de A.; MATTHEWS, S.; POWELL, A.A. The role of split seed coats in determining seed vigour in commercial seed lots of soybean, as measured by the electrical conductivity test. Seed Science and Technology, v.12, p.659-668, 1984.

PANOBIANCO, M.; VIEIRA, R.D. Electrical conductivity of soybean soaked seeds. I. Effect of genotype. Pesquisa Agropecuária Brasileira, v.31, n.9, p.621-7, 1996.

POWELL, A.A. Cell membranes and seed leachate conductivity in relation to the quality of seed for sowing. Journal of Seed Technology, v.10, n.2, p.81-100, 1986.

PRETE, C.E.C.; CICERO, S.M.; FOLEGATTI, M.V. Emergência de plântulas de soja no campo e sua relação com a embebição e condutividade elétrica das sementes. Semina, v.15, n.1, p.32-37, 1994.

RECH, E. G.; VILLELA, F.A.; TILLMANN, M.A.A. Avaliação rápida da qualidade fisiológica de sementes de ervilha. Revista Brasileira de Sementes, v.21, n.2, p.1-9, 1999.

ROSA, S.D.V.F.; VON-PINHO, E.V.R.; VIEIRA, M.G.G.C.; VEIGA, R.D. Eficácia do teste de condutividade elétrica para uso em estudos de danos de secagem em sementes de milho. Revista Brasileira de Sementes, v.22, n.1., p.54-63, 2000.

SÁ, M.E. Condutividade elétrica em sementes de tomate (Lycopersicon lycopersicum L.). Scientia Agricola, v.56, 
n.1, p.1-9, 1999.

SALINAS, A. R.; YODJIAN, A.M.; GRAVIOTTO, R.M.; BISARO, V. Pruebas de vigor y calidad fisiológica de semillas de soja. Pesquisa Agropecuária Brasileira, v.36, n.2, p.371-379, 2001.

SANTOS, M.R. . Qualidade fisiológica, sanidade e alterações bioquímicas em sementes de soja e suas relações com potencial de armazenamento e emergência no campo. 2004. 104f. Tese (Doutorado em Fitotecnia) Universidade Federal de Viçosa, Viçosa.

VANZOLINI, S.; NAKAGAWA, J. Teste de condutividade elétrica em sementes de amendoim: efeitos de temperatura e de período de embebição. Revista Brasileira de Sementes, v.21, n.1, p.41-45, 1999.

VIEIRA, R.D.; MINOHARA, L.; PANOBIANCO, M.; BERGAMASCHI, M.C.M.; MAURO, A.O. Comportamento de cultivares de soja quanto à qualidade fisiológica de sementes. Pesquisa Agropecuária Brasileira, v.33, n.2, p.123-130, 1998.

VIEIRA, R.D. PAIVA-AGUERO, J.A.; PERECIN, D. Electrical conductivity and field performance of soybean seeds. Seed Technology, v.21, n.1, p.15-24, 1999a.
VIEIRA, R.D.; PAIVA-AGUERO, J.A.; PERECIN, D.; BITTENCOURT, S.R.M. Correlation of electrical conductivity and other vigor tests with field emergence of soybean seedlings. Seed Technology, v.27, n.1, p.67-75, 1999b.

VIEIRA, R.D.; TEKRONY, D.M.; EGLI, D.B.; RUCKER, M. Electrical conductivity of soybean seeds after storage in several environments. Seed Science and Technology, v.29, p.599-6008, 2001.

VIEIRA, R.D. Teste de condutividade elétrica. In: VIEIRA, R.D.; CARVALHO, N.M.; SADER, R. Teste de vigor em sementes. Jaboticabal: FUNEP, 1994, p.103-32.

VIEIRA, R.D.; KRZYZANOWSKI, F.C. Teste de condutividade elétrica. In: KRZYZANOWSKI, F.C.; VIEIRA, R.D.; FRANÇA NETO, J.B. (Ed.). Vigor de sementes: conceitos e testes. Londrina: ABRATES, 1999. cap. 4, p.1-26.

YAKLICH, R.W.; KULIK, M.M.; ANDERSON, J.D. Evaluation of vigor tests in soybean seeds: relationship of ATP, conductivity, and radioactive tracer multiple criteria laboratory tests to field performance. Crop Science, v.19, n.6, p.806-810. 1979. 